\title{
Performance of High Indium Content InGaAs p-i-n Detector: A Simulation Study
}

\author{
Zhiwei Zhang, Guoqing Miao*, Hang Song, Hong Jiang, Zhiming Li, Dabing Li, Xiaojuan Sun, \\ Yiren Chen
}

State Key Laboratory of Luminescence and Applications, Changchun Institute of Optics, Fine Mechanics and Physics, Chinese Academy of Sciences, Changchun, China

Email: *miaogq@ciomp.ac.cn

Received 8 September 2015; accepted 13 November 2015; published 20 November 2015

\begin{abstract}
In this work, we investigate the performance of InGaAs p-i-n photodetectors with cut-off wavelengths near $2.6 \mu \mathrm{m}$. The influences of different substrate materials on the optoelectronic properties of InGaAs detector are also compared and discussed. GaAs-based device shows a significant enhancement in detector with a better performance for a InGaAs photodetector compared to InPbased device. In addition, our results show that the device performance is influenced by the conduction band offset. This work proves that InAlAs/InGaAs/GaAs structure is a promising candidate for high performance detector with optimally tuned band gap.
\end{abstract}

\section{Keywords}

InGaAs Detector, Near-Infrared, High Indium Content, Dark Current

\section{Introduction}

In recent years, there are growing needs for near-infrared detectors, the most important applications are spectral imaging, such as earth observation, remote sensing and environmental monitoring [1] [2]. Compared with the conventional HgCdTe or antimonide materials, the InGaAs material has higher electron mobility, better stability, anti-radiation characteristic, mature growth, device processing technology, and performance of the detectors, especially in high operation temperature and high irradiation environment. InGaAs p-i-n detector has recently become one of the most important near-infrared detectors. However, narrow detection range and large dark current remain a major problem restricting its development and application. There is a strong need for high performance extended wavelength InGaAs detector at room temperature.

Since the main limitation for device performance is associated with device structure, the design of novel device structures is an effective way to improve detector performance. In addition, the detailed characteristics of high indium content InGaAs detector, especially the dark current mechanism, have not been studied thoroughly. By increasing the In content of InGaAs, a quite large lattice mismatch between InGaAs epilayer and substrate is introduced and the dark current mechanism of the devices becomes complicated [3]. Therefore, more research is

\footnotetext{
*Corresponding author.
} 
needed on both device structure and dark current mechanism of InGaAs detector.

\section{Modeling Details}

The device simulation was done using APSYS, which self-consistently solves the Poisson's equation, and current continuity equations for electrons and holes within drift-diffusion model [4] [5]. Simulated structure is a modification of conventional InGaAs p-i-n photodetector reported in experiment [6]. From top to bottom, the epitaxial layer structure consists of a heavily p-doped cap layer, a slightly n-doped $\operatorname{In}_{0.82} \mathrm{Ga}_{0.18} \mathrm{As}$ adsorption layer, and a heavily n-doped substrate layer. Two types of substrates, InP and GaAs, were used in the simulation. Performance characteristics such as dark current, photocurrent, responsivity have been simulated at $300 \mathrm{~K}$. Analysis of the band diagrams can provide basic information about design correctness and device properties.

\section{Results and Discussion}

Figure 1 shows a comparison of the dark I-V characteristics of the two-type InGaAs photodetector and corresponding InP and GaAs substrates. It is found that the dark current of InP-based device is smaller than that of GaAs-based device at the first $0.1 \mathrm{~V}$. After $0.1 \mathrm{~V}$, the dark current of InP-based detector obviously increased. It is interesting that the GaAs substrate dark current becomes flat as the voltage increases from 0.1 to $0.5 \mathrm{~V}$. In the bias region, the simulated dark current is nearly independent of bias, and is sensitive primarily to the recombination velocity at the interface. As the reverse bias is increased to $0.5 \mathrm{~V}$, the dark current density increase to 175 $\mu \mathrm{A} / \mathrm{cm}^{2}$ and $110 \mu \mathrm{A} / \mathrm{cm}^{2}$, respectively. Figure 1 indicates that at room temperature and at a reverse bias of $0.5 \mathrm{~V}$, the dark current was reduced when GaAs act as substrate. This dark current is significantly lower than photodetector using InP substrate, whose tunneling component of dark current is substantial within this bias range. It was quite obvious that the reduction of the field-assisted tunneling process below the reverse bias of $0.5 \mathrm{~V}$ was responsible for this reduction in dark current for device.

The photocurrent characteristics for the InGaAs detector with different substrates are given in Figure 2. The relation between photocurrent and light power under a bias voltage of $0.5 \mathrm{~V}$ shows higher photocurrent with increasing light power as more light couples into the system. One can notice an increase of the photocurrent by using GaAs substrate. Excitation power and externally applied voltage are the same in both cases. Thus we attribute the improvement to the substrate. The in-depth electrical field is higher for the InAlAs/InGaAs/GaAs structures, and currents from the deeper layers go directly to the contacts, without crossing barriers of the heterostructure. Thus one can expect an increase in the photocurrent. Compared with the InP substrate, bigger photocurrent of the GaAs substrate structure is clearly demonstrated and explained especially for high-energy photon, which reveals that the substrate plays a key role in improving the quantum efficiency. It is observed that the GaAs-based detector has lower dark current density as well as a higher photocurrent compared to those of

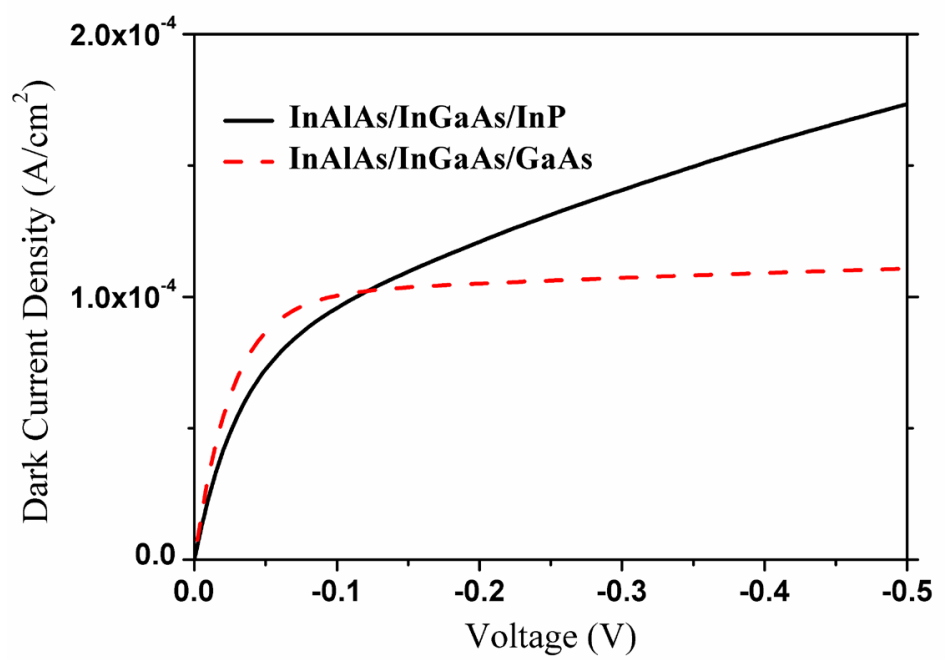

Figure 1. Dark current characteristics of InGaAs detectors with different substrates at room temperature. 


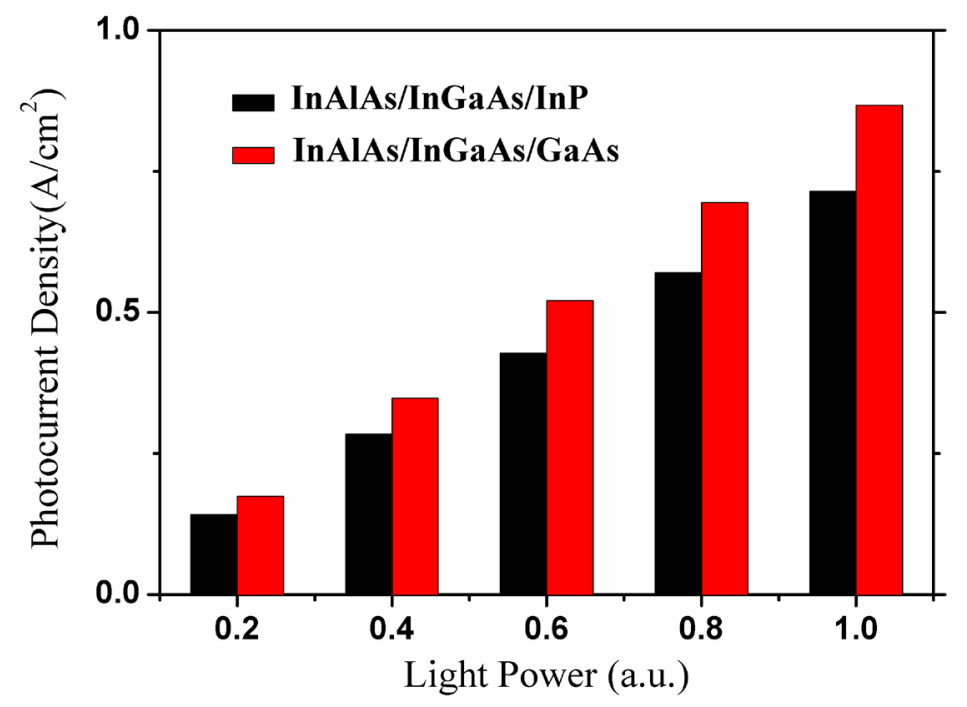

Figure 2. Photocurrent characteristics of InGaAs detectors with different substrates at room temperature.

InP-based detector. This meaning that there are better optoelectronic properties in the GaAs substrate and the performance are improved for GaAs-based detectors.

Figure 3 shows the measured relative response spectra of the extended wavelength InGaAs detectors with different substrate at $300 \mathrm{~K}$. The cut-off wavelength of InP-based detector is $2.5 \mu \mathrm{m}$, respectively. However, the cut-off wavelength shifts to $2.6 \mu \mathrm{m}$ when the substrate is GaAs. It is surprising that this GaAs substrate structure has a larger responsivity range compared with InP-based detector. According to simulation result, the wavelength become wide with GaAs substrate, which is due to the wavelength dependence in the Fermi-Dirac distribution function. Meanwhile, it should be mentioned that the measured responsibility of this InAlAs/InGaAs/InP system is still lower than InAlAs/InGaAs/GaAs. To understand this behavior qualitatively, it's worth noticing that the responsivity of a photodetector is the number of collected carriers per incident photon. When the substrate region is GaAs, the minority carriers switch to high mobility electrons as compared to the InP substrate sample where the minority carriers are holes. The rate that carriers leave the substrate region is faster in GaAs substrate samples as compared to InP substrate. Therefore, the probability of carrier recombination decreases and leads to higher responsivity. The long wavelength cutoff is not only determined by the absorption edge of the InGaAs layer, but aslo determined by the substrate.

The calculated energy band structure of the proposed detector around the InP and GaAs substrate layers at a bias voltage near the onset condition without illumination is shown in Figure 4. A broadband absorption enhancement is obtained by combining the phenomena, and the dark current can be tuned by changing substrate. This is due to high electron mobility in InGaAs and to the large conduction band discontinuity between absorption and substrate layers which favors charge transfer and confinement in the channel. Compared to the major industrial competitors, the InP-based device, GaAs-based device has a higher conduction band offset, which provides good electron confinement [7] [8]. The energy states near the band edge are occupied and results a decrease in the generation rate and related dark current. Because it can have a high conduction band offset region at the interface, the GaAs-based detector may be less affected by misfit dislocations than InP-based with low offset region, which exhibit low leakage current when significant numbers of such misfit dislocations intercept the interface region.

\section{Conclusion}

A novel design, InAlAs/InGaAs/GaAs structure, with a great potential for near-infrared detection was found in this research. After optimizing, the wavelength response up to $2.6 \mu \mathrm{m}$ at room temperature. The results show that the performance of InGaAs detector strongly depend on the device structure, i.e., substrate material. In addition, research results demonstrate that the conduction band offset of heteojunction interface has important 


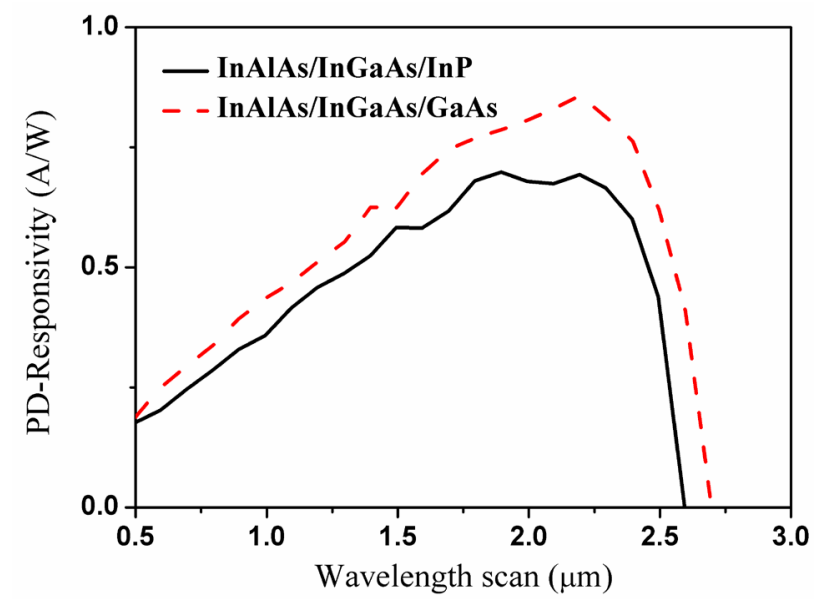

Figure 3. Relative spectral response of InGaAs detectors with different substrates at bias $-0.5 \mathrm{~V}$.

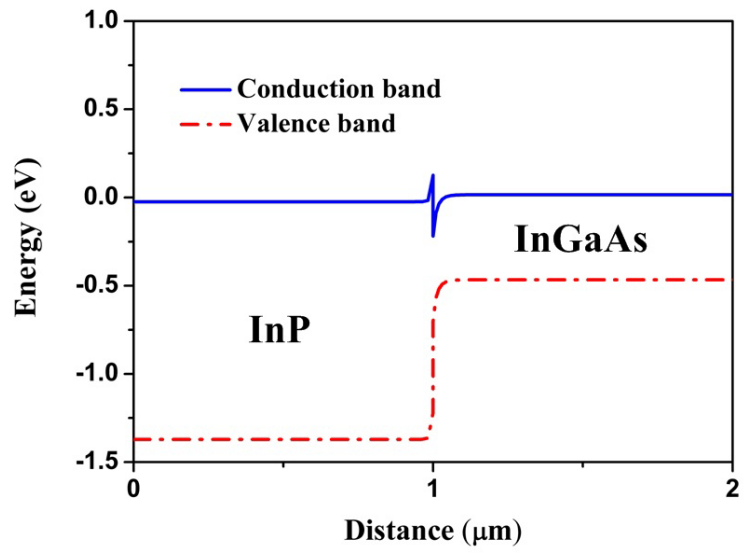

(a) InAlAs/InGaAs/InP

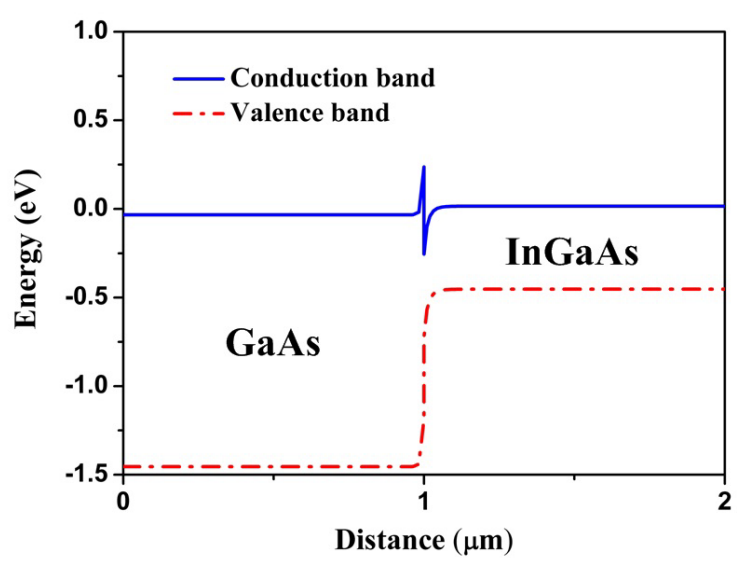

(b) InAlAs/InGaAs/GaAs

Figure 4. Energy band structure for the InGaAs photodetector with different substrates for room temperature condition.

influence on the movement of carrier, which could result in changes of detector performance. The higher conduction band offset is more favorable for the low dark current operations.

\section{Acknowledgements}

This work is supported by the National Key Basic Research Program of China (No. 2012CB619201), and the Jilin Provincial Science and Technology Department (No. 20140520117JH).

\section{References}

[1] Hoogeveen, R.W.M., van der A, R.J. and Goede, A.P.H. (2001) Extended Wavelength InGaAs Infrared (1.0-2.4 $\mu \mathrm{m})$ Detector Arrays on SCIAMACHY for Space-Based Spectrometry of the Earth Atmosphere. Infrared Phys. Technol., 42, 1-16. http://dx.doi.org/10.1016/S1350-4495(00)00061-X

[2] Zhang, K.F., Tang, H.J., Wu, X.L., Li, Y.F., Li, T., Li, X. and Gong, H.M. (2009) Effects of an Anodic Oxide Passivation Layer on Mesa-Type InGaAs (PIN) Photodetectors. Semicond. Sci. Technol., 24, 015008. http://dx.doi.org/10.1088/0268-1242/24/1/015008

[3] Gu, Y., Wang, K., Li, C., Fang, X., Cao, Y.Y. and Zhang, Y.G. (2011) High Indium Content InGaAs Photodetector: with InGaAs or InAlAs Graded Buffer Layers. J. Infrared Millimeter Waves, 30, 481-485. http://dx.doi.org/10.3724/SP.J.1010.2011.00481

[4] Anderson, C.R. (2009) Efficient Solution of the Schroedinger-Poisson Equations in Layered Semiconductor Devices. J. 
Comput. Phys., 228, 4745. http://dx.doi.org/10.1016/j.jcp.2009.03.037

[5] Marquardt, O., Boeck, S., Freysoldt, C., Hickel, T., Schulz, S., Neugebauer, J. and O’Reilly, E.P. (2014) A Generalized Plane-Wave Formulation of k·p Formalism and Continuum-Elasticity Approach to Elastic and Electronic Properties of Semiconductor Nanostructures. Comput. Mater. Sci., 95, 280-287. http://dx.doi.org/10.1016/j.commatsci.2014.06.047

[6] Miao, G.Q., Zhang, T.M., Zhang, Z.W. and Jin, Y.X. (2013) Extended Spectral Response in $\operatorname{In}_{0.82} \mathrm{Ga}_{0.18} \mathrm{As} / \mathrm{InP}$ Photodetector Using InP as a Window Layer Grown by MOCVD. Cryst Eng Comm, 15, 8461-8464. http://dx.doi.org/10.1039/c3ce41162a

[7] Galluppi, M., Geelhaar, L. and Riechert, H. (2006) Band Offsets Analysis of Dilute Nitride Single Quantum Well Structures Employing Surface Photo Voltage Measurements. J. Electron. Mater., 35, 733-737. http://dx.doi.org/10.1007/s11664-006-0130-0

[8] Chen, X.Y., Zhang, Y.G., Gu, Y., Zhou, L., Cao, Y.Y., Fang, X. and Li, H. (2014) GaAs-Based $\operatorname{In}_{0.83} \mathrm{Ga}_{0.17} \mathrm{As}$ Photodetector Structure Grown by Gas Source Molecular Beam Epitaxy. J. Cryst. Growth, 393, 75-80.

http://dx.doi.org/10.1016/j.jcrysgro.2013.11.083 